\title{
JUNO Physics Prospects
}

\section{João Pedro Athayde Marcondes de André ${ }^{a, *}$ on behalf of the JUNO Collaboration} (a complete list of authors can be found at the end of the proceedings)

${ }^{a} I P H C$, Université de Strasbourg, CNRS/IN2P3, F-67037 Strasbourg, France

E-mail: jpandre@iphc.cnrs.fr

JUNO is a multi-purpose underground neutrino observatory being constructed in the south of China. The main detector, with a 20 kton liquid scintillator target instrumented with about 18k 20" PMT and about 26k 3" PMT, will be strategically located $53 \mathrm{~km}$ from the Taishan and Yangjiang Nuclear Power Plants. Using reactor antineutrinos, JUNO will be able to measure several neutrino oscillation parameters with sub-percent precision as well as to determine the neutrino mass ordering to $\sim 3 \sigma$ over 6 years of operation. Furthermore, JUNO will have a broad physics program, ranging from studying neutrinos from other sources, such as solar and supernova neutrinos, to searching for BSM physics such as proton decay. This talk will give an overview on the JUNO's broad physics potential.

$37^{\text {th }}$ International Cosmic Ray Conference (ICRC 2021)

July 12th-23rd, 2021

Online - Berlin, Germany

${ }^{*}$ Presenter 


\section{Introduction}

Since the discovery of neutrino oscillations at the end of the last century $[1,2]$ which demonstrated neutrinos are massive particles, significant progress has been made in determining their properties [3]. In order to describe 3 flavor neutrino oscillations, 6 independent parameters are required in total: 2 mass squared differences $\left(\Delta m_{21}^{2} \text {, and } \Delta m_{32}^{2} \text { or } \Delta m_{31}^{2}\right)^{1}, 3$ mixing angles $\left(\theta_{12}\right.$, $\theta_{13}$, and $\left.\theta_{23}\right)$, and a CP-violating phase $\left(\delta_{\mathrm{CP}}\right)$. At this moment, most of these parameters have been measured to a $\lesssim 5 \%$ precision with the exception of the sign of $\Delta m_{32}^{2}$ and the value of $\delta_{\mathrm{CP}}[4,5]$. The unknown sign of $\Delta m_{32}^{2}$ creates two possible different neutrino mass orderings (NMOs), which are named "normal ordering" (when $m_{1}<m_{2}<m_{3}$, with $m_{i}$ being the mass associated with the neutrino mass eigenstate $v_{i}$ ) and "inverted ordering" (when $m_{3}<m_{1}<m_{2}$ ). The "inverted" name refers to the fact that in this case the $v_{e}$ effective mass is not the smallest, as would have been normally expected given the masses of the other fermions of the Standard Model of Particle Physics.

With the discovery of a rather large $\theta_{13}$ value in 2012 [6], it became possible to consider using medium baseline reactor experiments to determine the NMO. The Jiangmen Underground Neutrino Observatory (JUNO) was born of this idea, and then developed to cover a broad physics program including studying neutrinos from natural sources sources such as solar, supernova, and atmospheric neutrinos, and to search for physics beyond the Standard Model of Particle Physics.

This document relies heavily on Refs. [7, 8]. After an initial description of JUNO in Sec. 2, this proceedings will focus on the NMO measurement in Sec. 3, the precision measurement of oscillation parameters in Sec. 4, and on studies using atmospheric neutrinos in Sec. 5. Studies with JUNO involving supernova neutrinos, the diffuse supernova background, and solar neutrinos are also covered in separate proceedings of this conference [9-11].

\section{JUNO}

The JUNO detector is located in the south-east of China at a distance of $53 \mathrm{~km}$ from the Yangjiang and Taishan Nuclear Power Plants (NPP). This location was selected to optimize the NMO sensitivity using reactor neutrinos, requiring the detector to be placed at the first $\bar{v}_{e}$ disappearance maximum that is driven by $\Delta m_{21}^{2}$ while measuring simultaneously the oscillation pattern from both $\Delta m_{32}^{2}$ and $\Delta m_{21}^{2}$. JUNO is currently being constructed and is expected to start taking data in 2022.

The JUNO detector is composed of three main parts, as shown in Fig. 1: the Central Detector (CD), the Water Cherenkov Detector (WCD) and the Top Tracker (TT). The CD is composed of a $35.4 \mathrm{~m}$ diameter acrylic sphere containing $20 \mathrm{kton}$ of liquid scintillator. This sphere is monitored by $18 \mathrm{k} 20$ " and 26k 3" photomultiplier tubes (PMT) that surround the acrylic sphere. High PMT coverage and high light yield liquid scintillator are required for the JUNO CD to reach a $3 \%$ energy resolution at $1 \mathrm{MeV}$, required for the NMO determination. The WCD is a cylinder of diameter $43.5 \mathrm{~m}$ and height $44 \mathrm{~m}$ surrounding the $\mathrm{CD}$. This volume is filled with $35 \mathrm{kton}$ of ultra-pure water and instrumented with 2.4k 20" PMTs with the goal of tracking atmospheric muons entering the detector and protecting the CD from external radioactivity. The TT, located on top of the WCD,

\footnotetext{
${ }^{1} \Delta m_{32}^{2}$ and $\Delta m_{31}^{2}$ are not independent as $\Delta m_{31}^{2}=\Delta m_{32}^{2}+\Delta m_{21}^{2}$
} 


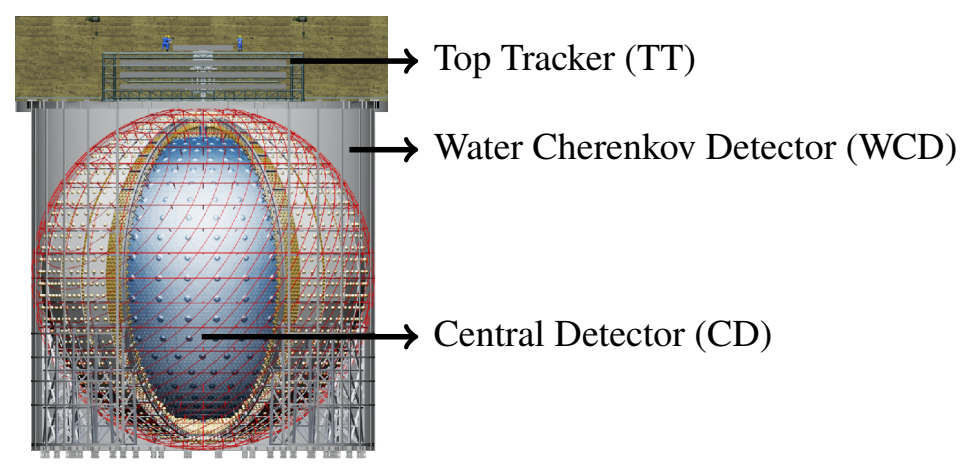

Figure 1: The JUNO detector.

is made of 3 layers of plastic scintillator used to precisely track some of the atmospheric muons entering the detector. The TT covers about $60 \%$ of the surface above the WCD.

In JUNO, reactor electron anti-neutrinos will be detected using the inverse beta decay (IBD) reaction: $\bar{v}_{\mathrm{e}}+\mathrm{p} \rightarrow \mathrm{n}+\mathrm{e}^{+}$. The positron produced in this reaction, which will keep most of the electron anti-neutrino energy, will quickly deposit most of its energy and annihilate with electrons in the medium producing a pair of $511 \mathrm{keV}$ gamma-rays. The neutron produced in this reaction, will be captured by a proton after a mean time of about $200 \mu$ s, and its de-excitation will produce a $2.2 \mathrm{MeV}$ gamma-ray. The temporal and spatial coincidence signature created by these prompt (positron) and delayed (neutron) signals is characteristic of the IBD and is essential to suppress a large fraction of the background. Given in the IBD the positron keeps most of the neutrino energy, the reconstructed prompt energy is used to determine the electron anti-neutrino energy required for oscillation studies.

Due to the lack of a reference reactor electron anti-neutrino spectrum with a similar resolution to the JUNO detector, the JUNO-TAO detector [12], shown in Fig. 2, was added to the project. The JUNO-TAO detector is located $30 \mathrm{~m}$ from one of the Taishan NPP reactor cores. With a surface $10 \mathrm{~m}^{2}$ of silicon photomultipliers panels operated at $-50^{\circ} \mathrm{C}$ monitoring a 1 ton fiducial volume containing Gd-loaded liquid scintillator, JUNO-TAO will provide an energy spectrum for reactor neutrinos with an energy resolution of less than $2 \%$ at $1 \mathrm{MeV}$ which is better than that of JUNO. This reference spectrum will effectively reduce the impact of possible unknown fine-structures in this spectrum [13] on the measurement of neutrino oscillations.

\section{Measuring the Neutrino Mass Ordering}

The neutrino flux from the Taishan and Yangjiang NPPs will be detected in the JUNO detector as shown in Fig. 3, as a function of the true neutrino energy. In this figure the different oscillation patterns, arising from the $\Delta m_{21}^{2}$ and $\Delta m_{32}^{2}$ oscillation frequencies, can be clearly identified. The slow oscillation, tied to $\Delta m_{21}^{2}$, shows a single large deficit in the spectrum with a maximum around $3 \mathrm{MeV}$, but that spans the entire energy range. The fast oscillation, tied to $\Delta m_{32}^{2}$, produces wiggles in the spectrum over the entire range, but with a much smaller amplitude. The position of these wiggles depends on the neutrino mass ordering and it is trough their measurement that JUNO determines the NMO. It is worth noting that in Fig. 3 the true neutrino energy spectra are shown. 


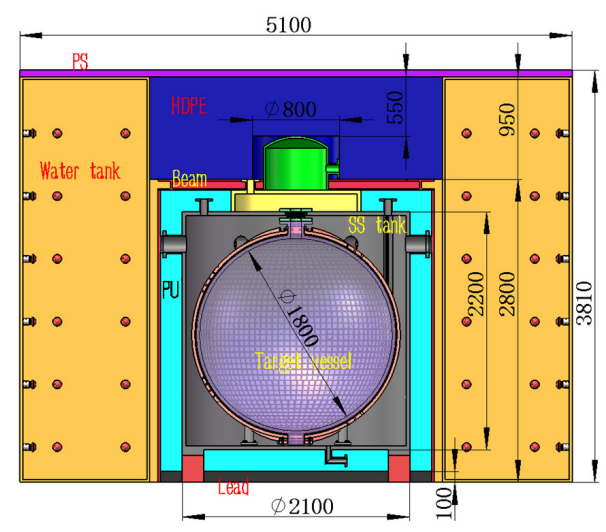

Figure 2: The JUNO-TAO detector.

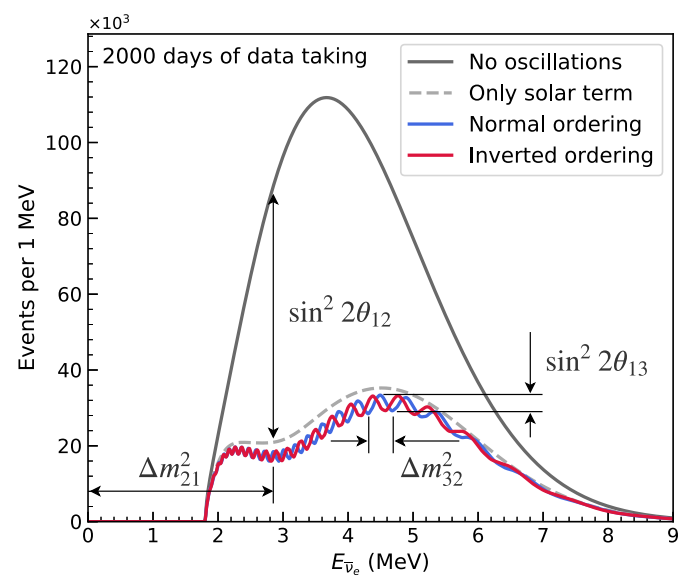

Figure 3: Reactor electron anti-neutrino spectra in JUNO as a function of the neutrino energy. From Ref. [8].

Once the energy resolution of the detector is taken into account the fast wiggles at the lower part of the energy spectrum will no longer be distinguishable, and the measurement will rely mainly on those at higher energies. This is the reason why the detector energy resolution is one of the key parameters towards the measurement of the NMO.

Since Ref. [7], several changes impacted the project with opposing impacts to the NMO analysis [8]. On one hand, only 2 of the 4 originally planned Taishan NPP reactor cores were built. The plans to build the other 2 cores are currently uncertain. On the other hand, the PMT quantum efficiency and the measured light scintillator light yield were higher than considered in Ref. [7]. In addition to these changes, the unoscillated reactor spectrum will now be better constrained than was expected in Ref. [7] thanks to JUNO-TAO. These changes both increase and decrease the JUNO NMO sensitivity and are expected in the end to have a small net impact in the final JUNO sensitivity. Detailed analyses are currently ongoing to provide updated sensitivities, taking into account not only the aforementioned changes but also a more realistic description of the detector and calibration based on measurements done by the collaboration. The discussion on the remainder of this section and on the next section will be done based on Ref. [7].

The sensitivity of JUNO to the NMO is calculated using an Asimov sample. Fits to both 


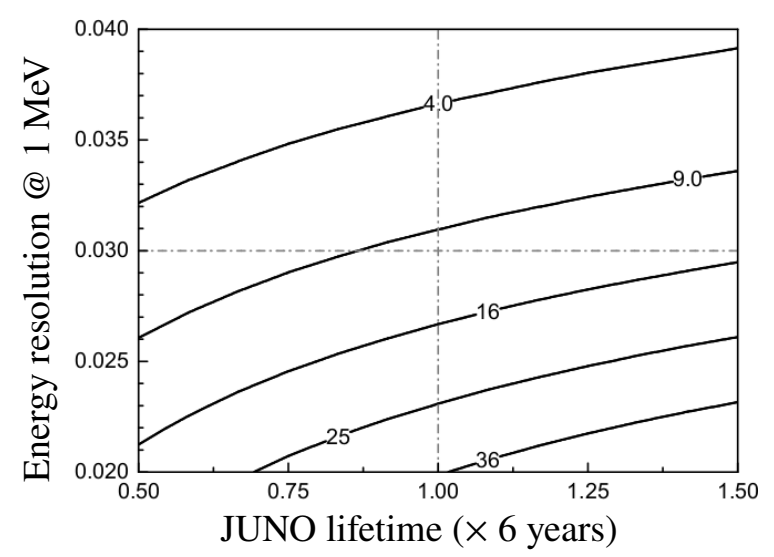

Figure 4: JUNO sensitivity to the NMO ( $\Delta \chi^{2}$ contours) as a function of the JUNO lifetime scaled from 6 years and the energy resolution at $1 \mathrm{MeV}$. From Ref. [7].

orderings are performed and the difference between minimum $\chi^{2}$ in the true and false orderings is calculated and noted as $\Delta \chi^{2}$. For 6 years of data taking, a $\Delta \chi^{2}=16$ is expected considering only statistical errors assuming all reactors cores are located at exactly the same optimal distance of $\sim 52.5 \mathrm{~km}$. After taking into account the real distances to each reactor cores ${ }^{2}$ along with adding systematic errors on the signal and background, the JUNO NMO sensitivity is reduced to $\Delta \chi^{2}=10$. In Fig. 4 is shown the dependency of the $\Delta \chi^{2}$ value obtained as a function of the luminosity, scaled from the 6 years baseline, and the energy resolution at $1 \mathrm{MeV}$. It highlights the importance of achieving the previously discussed $3 \%$ energy resolution as even increasing by $50 \%$ the amount of data, JUNO is not able to reach $3 \sigma$ sensitivity (ie, $\Delta \chi^{2}=9$ ) for a $3.5 \%$ energy resolution. To reach this goal, the JUNO detector uses 4 complementary calibration systems. A detailed calibration strategy for JUNO is presented in Ref. [14], where a (3.02 \pm 0.01$) \%$ energy resolution at $1 \mathrm{MeV}$ and a $(0.03 \pm 0.01) \%$ energy bias are achieved in the baseline detector configuration.

In the previously discussed JUNO sensitivity, no external data is used to constrain the $\Delta m_{32}^{2}$ value fitted by JUNO. By using an external $1 \%$ constraint from $v_{\mu}$ disappearance measurements, the NMO sensitivity can be improved to $4 \sigma$ [7]. This is possible thanks to the intrinsic difference in the $\bar{v}_{e} \rightarrow \bar{v}_{e}$ and $v_{\mu} \rightarrow v_{\mu}$ oscillations which lead to different best-fit values for $\Delta m_{32}^{2}$ when fitting the wrong ordering. In addition to simply adding a prior from $v_{\mu}$ disappearance measurements, several analysis performed combining JUNO with accelerator [15] and atmospheric [16-18] neutrino experiments have highlighted the possibility of boosting the NMO sensitivity to $5 \sigma$.

\section{Precision Measurement of Oscillation Parameters}

Using the same sample used for the NMO measurement, it is also possible to determine the values of 4 of the oscillation parameters. The JUNO baseline is ideal for precision measurements of the $\Delta m_{21}^{2}$ and $\theta_{12}$ parameters being located in the first $\bar{v}_{e}$ disappearance peak from $\Delta m_{21}^{2}$. Additionally, the $3 \%$ energy resolution will make it possible to measure several $\Delta m_{32}^{2}$ oscillations, enabling JUNO to achieve sub-percent precision on this parameter. While JUNO can also measure $\theta_{13}$, it is

\footnotetext{
${ }^{2}$ Including also the Daya-Bay and Huizhou NPP cores which are located at significantly longer baselines.
} 
not expected to achieve the precision of current reactor experiments. Fig. 5 shows JUNO's expected precision on $\Delta m_{21}^{2}, \Delta m_{e e}^{2}$, and $\sin ^{2} \theta_{12}$ as a function of the energy resolution. The $\Delta m_{e e}^{2}$ parameter used in this study is a proxy for $\Delta m_{32}^{2}$ and is defined as: $\Delta m_{e e}^{2}=\cos ^{2} \theta_{12} \Delta m_{31}^{2}+\sin ^{2} \theta_{12} \Delta m_{32}^{2}$. For the precision measurement of the neutrino oscillation parameters, the energy resolution requirement has a significantly smaller impact, and for all studied energy resolutions JUNO is expected to reach a better than $0.6 \%$ precision on these 3 parameters. The changes since Ref. [7] that impacted the NMO analysis will also have an impact in these precision measurements. Taking into account these changes, a reassessment of JUNO's precision to measure these 3 parameters is in progress, although the results are not expected to change significantly.

\section{Atmospheric Neutrinos}

As discussed previously, besides measuring reactor neutrinos JUNO will also measure neutrinos from other sources. Among these sources are neutrinos produced in the showers originated by cosmic-rays interacting in the Earth's atmosphere. Atmospheric neutrinos have a long history of being used to study neutrino oscillations, since their discovery in 1998 by Super-Kamiokande [1]. More recently, atmospheric neutrino experiments have also been proposed to determine the NMO [19, 20] using matter effects during the neutrino propagation through the Earth. While the JUNO detector is not optimized to measure atmospheric neutrinos, the JUNO sensitivity to NMO using atmospheric neutrinos is expected to be between $0.9 \sigma$ and $1.8 \sigma$ with 10 years of data, depending on the assumptions regarding the detector capability to identify and reconstruct atmospheric neutrinos [7]. Besides a direct NMO measurement, studies are also ongoing to combine the sensitivity to the NMO using reactor and atmospheric neutrinos within JUNO.

In addition to being used to study neutrino oscillations, JUNO will also be able to measure the $v_{e}+\bar{v}_{e}$ and $v_{\mu}+\bar{v}_{\mu}$ spectrum between $100 \mathrm{MeV}$ and $10 \mathrm{GeV}$, as shown in Fig. 6 . In this analysis, the different hit time patterns of electron and muon neutrinos, caused by the creation of an electron or muon in the neutrino charged current interaction, are used to discriminate the flavor of the detected neutrinos. Given the higher energy of these neutrinos in comparison to reactor neutrinos,

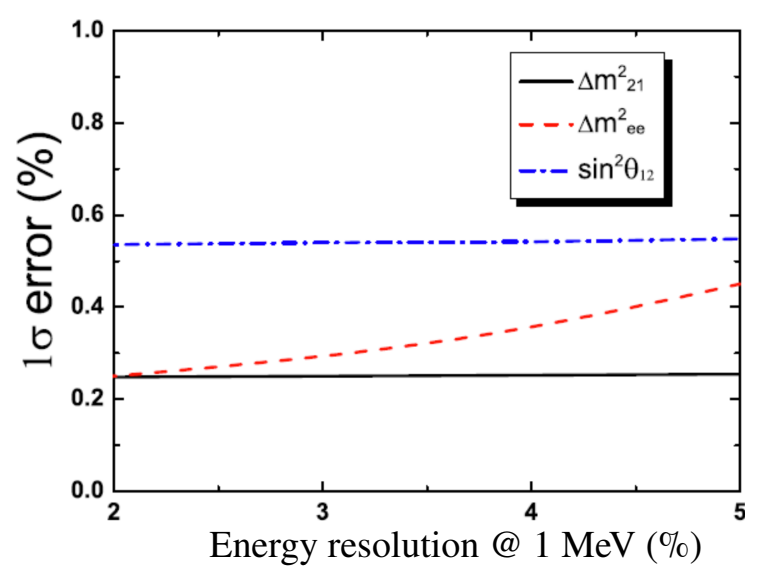

Figure 5: Expected precision on $\Delta m_{21}^{2}, \Delta m_{e e}^{2}$ (used as a proxy for $\Delta m_{32}^{2}$ ), and $\sin ^{2} \theta_{12}$ as a function of the energy resolution. From Ref. [7]. 


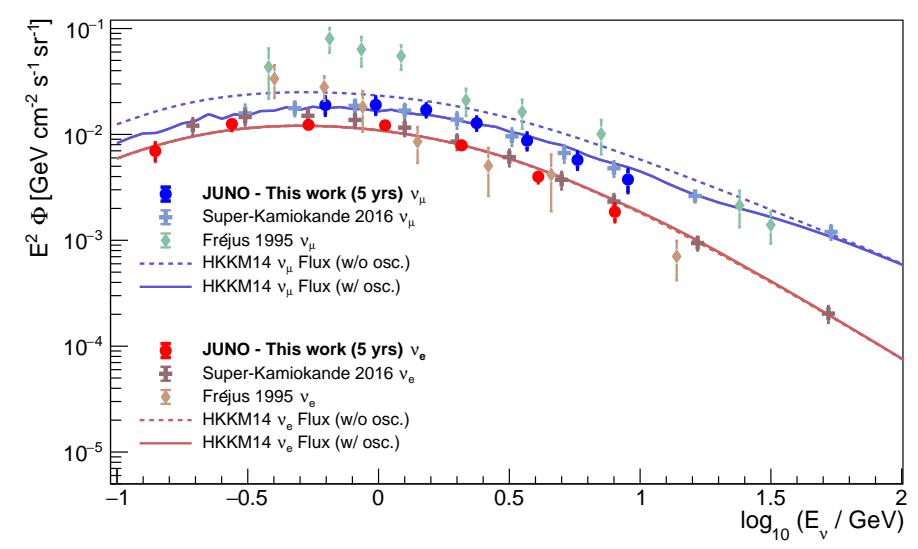

Figure 6: Atmospheric neutrino energy spectra reconstructed for JUNO for $v_{\mu}$ (blue) and $v_{e}$ (red). From Ref. [8].

the 3" PMT system was used primarily to measure these time patterns as its Transit Time Spread of the order of the nanosecond is smaller than that for most 20" PMTs in JUNO. To guarantee a good quality of the energy reconstruction, events close to the detector boundary and partially contained events are rejected. An unfolding method is then used in a Monte Carlo sample to obtain the atmospheric neutrino spectra shown in Fig. 6. The final uncertainties in these unfolded spectra are between $10 \%$ and $25 \%$ with 5 years of data, showing a great potential of the detector in the atmospheric low energy region. More details about this analysis can be found in Ref. [21].

\section{Other Physics Topics in JUNO}

In addition to other topics covered in other proceedings in this conference, such as supernova neutrinos [9], diffuse supernova background neutrinos [10], and solar neutrinos [11], JUNO has the potential to address other open questions in a wide range of domains. For example, JUNO will be able to measure the geo-neutrino flux to about 5\% precision in 10 years, which can then be compared to the expectation from geological surveys and used to test geological models. In these models, geo-neutrino measurements estimate the abundance of $U$ and $T h$ in the Earth, and, with that, the Earth's heat flow coming from radioactive sources. JUNO will be able to probe Beyond Standard Model physics by looking for nucleon decay, in particular, via the channel $\mathrm{p} \rightarrow \mathrm{K}^{+}+\bar{v}$. In this particular channel, JUNO would observe a triple coincidence signature that significantly helps to reject background, and makes it possible for JUNO's sensitivity to reach $8.3 \times 10^{33}$ years (90\% C.L.) with 10 years of data. More details on these studies are available in Refs. [7, 8].

\section{References}

[1] Y. Fukuda et al. (Super-Kamiokande Collaboration). "Evidence for oscillation of atmospheric neutrinos". Phys. Rev. Lett., 81:1562-1567, 1998.

[2] Q. R. Ahmad et al. (SNO Collaboration). "Direct evidence for neutrino flavor transformation from neutral current interactions in the Sudbury Neutrino Observatory". Phys. Rev. Lett., 89:011301, 2002. 
[3] P. A. Zyla et al. (Particle Data Group Collaboration). "Review of Particle Physics". PTEP, 2020(8):083C01, 2020.

[4] I. Esteban et al. "The fate of hints: updated global analysis of three-flavor neutrino oscillations". JHEP, 09:178, 2020.

[5] P. F. de Salas et al. "2020 global reassessment of the neutrino oscillation picture". JHEP, 02:071, 2021.

[6] F. P. An et al. (Daya Bay Collaboration). "Observation of electron-antineutrino disappearance at Daya Bay". Phys. Rev. Lett., 108:171803, 2012.

[7] F. An et al. (JUNO Collaboration). "Neutrino Physics with JUNO”. J. Phys. G, 43(3):030401, 2016.

[8] A. Abusleme et al. (JUNO Collaboration). "JUNO Physics and Detector". arXiv:2104.02565, 2021.

[9] X. Huang (JUNO Collaboration). "Potential of Core-Collapse Supernova Neutrino Detection at JUNO". PoS(ICRC2021)1076, 2021.

[10] J. Cheng (JUNO Collaboration). "Diffuse Supernova Neutrino Background Detection at JUNO". PoS(ICRC2021)1187, 2021.

[11] J. Zhao (JUNO Collaboration). "Feasibility of detecting B8 solar neutrinos at JUNO". PoS(ICRC2021)1229, 2021.

[12] A. Abusleme et al. (JUNO Collaboration). "TAO Conceptual Design Report: A Precision Measurement of the Reactor Antineutrino Spectrum with Sub-percent Energy Resolution". arXiv:2005.08745, 2020.

[13] D. A. Dwyer et al. "Spectral Structure of Electron Antineutrinos from Nuclear Reactors". Phys. Rev. Lett., 114(1):012502, 2015.

[14] A. Abusleme et al. (JUNO Collaboration). "Calibration Strategy of the JUNO Experiment". JHEP, 03:004, 2021.

[15] A. Cabrera et al. "Earliest Resolution to the Neutrino Mass Ordering?". arXiv:2008.11280, 2020.

[16] M. Blennow et al. "Determination of the neutrino mass ordering by combining PINGU and Daya Bay II". JHEP, 09:089, 2013.

[17] M. Aartsen et al. (JUNO Collaboration members and IceCube Gen2 Collaboration). "Combined sensitivity to the neutrino mass ordering with JUNO, the IceCube Upgrade, and PINGU". Phys. Rev. D, 101(3):032006, 2020.

[18] J. P. A. M. de André (KM3NeT Collaboration and members of the JUNO Collaboration). "Neutrino mass ordering determination through combined analysis with JUNO and KM3NeT/ORCA". PoS(ICRC2021)1196, 2021.

[19] M. G. Aartsen et al. (IceCube-PINGU Collaboration). "Letter of Intent: The Precision IceCube Next Generation Upgrade (PINGU)”. arXiv:1401.2046, 2014.

[20] S. Adrian-Martinez et al. (KM3Net Collaboration). "Letter of intent for KM3NeT 2.0". J. Phys. G, 43(8):084001, 2016.

[21] A. Abusleme et al. (JUNO Collaboration). "Measuring low energy atmospheric neutrino spectra with the JUNO detector". arXiv:2103.09908, 2021. 


\section{Full Authors List: JUNO Collaboration}

Angel Abusleme ${ }^{5}$, Thomas Adam ${ }^{45}$, Shakeel Ahmad ${ }^{66}$, Rizwan Ahmed ${ }^{66}$, Sebastiano Aiello ${ }^{55}$, Muhammad Akram ${ }^{66}$, Fengpeng $\mathrm{An}^{29}$, Qi An ${ }^{22}$, Giuseppe Andronico ${ }^{55}$, Nikolay Anfimov ${ }^{67}$, Vito Antonelli ${ }^{57}$, Tatiana Antoshkina ${ }^{67}$, Burin Asavapibhop ${ }^{71}$, João Pedro Athayde Marcondes de André ${ }^{45}$, Didier Auguste ${ }^{43}$, Andrej Babic ${ }^{70}$, Nikita Balashov ${ }^{67}$, Wander Baldini ${ }^{56}$, Andrea Barresi ${ }^{58}$, Davide Basilico ${ }^{57}$, Eric Baussan ${ }^{45}$, Marco Bellato ${ }^{60}$, Antonio Bergnoli ${ }^{60}$, Thilo Birkenfeld ${ }^{48}$, Sylvie Blin ${ }^{43}$, David Blum ${ }^{54}$, Simon Blyth $^{40}$, Anastasia Bolshakova ${ }^{67}$, Mathieu Bongrand ${ }^{47}$, Clément Bordereau ${ }^{44,40}$, Dominique Breton ${ }^{43}$, Augusto Brigatti ${ }^{57}$, Riccardo Brugnera $^{61}$, Riccardo Bruno ${ }^{55}$, Antonio Budano ${ }^{64}$, Mario Buscemi ${ }^{55}$, Jose Busto ${ }^{46}$, Ilya Butorov ${ }^{67}$, Anatael Cabrera ${ }^{43}$, $\mathrm{Hao} \mathrm{Cai}^{34}$, Xiao Cai ${ }^{10}$, Yanke Cai ${ }^{10}$, Zhiyan $\mathrm{Cai}^{10}$, Riccardo Callegari ${ }^{61}$, Antonio Cammi ${ }^{59}$, Agustin Campeny ${ }^{5}$, Chuanya $\mathrm{Cao}^{10}$, Guofu $\mathrm{Cao}^{10}$, Jun $\mathrm{Cao}^{10}$, Rossella Caruso ${ }^{55}$, Cédric Cerna ${ }^{44}$, Jinfan Chang ${ }^{10}$, Yun Chang ${ }^{39}$, Pingping Chen ${ }^{18}$, Po-An Chen ${ }^{40}$, Shaomin Chen ${ }^{13}$, Xurong Chen ${ }^{26}$, Yi-Wen $\mathrm{Chen}^{38}$, Yixue $\mathrm{Chen}^{11}$, Yu Chen ${ }^{20}$, Zhang Chen ${ }^{10}$, Jie Cheng ${ }^{10}$, Yaping Cheng ${ }^{7}$, Alexey Chetverikov $^{67}$, Davide Chiesa ${ }^{58}$, Pietro Chimenti ${ }^{3}$, Artem Chukanov ${ }^{67}$, Gérard Claverie ${ }^{44}$, Catia Clementi ${ }^{62}$, Barbara Clerbaux ${ }^{2}$, Selma Conforti Di Lorenzo ${ }^{44}$, Daniele Corti ${ }^{60}$, Flavio Dal Corso ${ }^{60}$, Olivia Dalager ${ }^{74}$, Christophe De La Taille ${ }^{44}$, Jiawei Deng ${ }^{34}$, Zhi Deng ${ }^{13}$, Ziyan Deng ${ }^{10}$, Wilfried Depnering ${ }^{52}$, Marco Diaz ${ }^{5}$, Xuefeng Ding ${ }^{57}$, Yayun Ding ${ }^{10}$, Bayu Dirgantara ${ }^{73}$, Sergey Dmitrievsky $^{67}$, Tadeas Dohnal ${ }^{41}$, Dmitry Dolzhikov ${ }^{67}$, Georgy Donchenko ${ }^{69}$, Jianmeng Dong ${ }^{13}$, Evgeny Doroshkevich ${ }^{68}$, Marcos $\operatorname{Dracos}^{45}$, Frédéric Druillole ${ }^{44}$, Ran Du ${ }^{10}$, Shuxian Du ${ }^{37}$, Stefano Dusini ${ }^{60}$, Martin Dvorak ${ }^{41}$, Timo Enqvist ${ }^{42}$, Heike Enzmann ${ }^{52}$, Andrea Fabbri ${ }^{64}$, Lukas Fajt ${ }^{70}$, Donghua Fan ${ }^{24}$, Lei Fan ${ }^{10}$, Jian Fang ${ }^{10}$, Wenxing Fang ${ }^{10}$, Marco Fargetta ${ }^{55}$, Dmitry Fedoseev ${ }^{67}$, Vladko Fekete ${ }^{70}$, Li-Cheng Feng ${ }^{38}$, Qichun Feng ${ }^{21}$, Richard Ford ${ }^{57}$, Amélie Fournier ${ }^{44}$, Haonan Gan ${ }^{32}$, Feng Gao ${ }^{48}$, Alberto Garfagnini $^{61}$, Arsenii Gavrikov ${ }^{61}$, Marco Giammarchi ${ }^{57}$, Agnese Giaz ${ }^{61}$, Nunzio Giudice ${ }^{55}$, Maxim Gonchar ${ }^{67}$, Guanghua Gong ${ }^{13}$, Hui Gong ${ }^{13}$, Yuri Gornushkin ${ }^{67}$, Alexandre Göttel ${ }^{50,48}$, Marco Grassi ${ }^{61}$, Christian Grewing ${ }^{51}$, Vasily Gromov ${ }^{67}$, Minghao Gu ${ }^{10}$, Xiaofei $\mathrm{Gu}^{37}$, Yu Gu${ }^{19}$, Mengyun Guan ${ }^{10}$, Nunzio Guardone ${ }^{55}$, Maria Gul ${ }^{66}$, Cong Guo ${ }^{10}$, Jingyuan Guo ${ }^{20}$, Wanlei Guo ${ }^{10}$, Xinheng Guo ${ }^{8}$, Yuhang Guo ${ }^{35,50}$, Paul Hackspacher ${ }^{52}$, Caren Hagner ${ }^{49}$, Ran Han ${ }^{7}$, Yang Hann ${ }^{20}$, Muhammad Sohaib Hassan ${ }^{66}$, Miao $\mathrm{He}^{10}$, Wei He${ }^{10}$, Tobias Heinz ${ }^{54}$, Patrick Hellmuth ${ }^{44}$, Yuekun Heng ${ }^{10}$, Rafael Herrera ${ }^{5}$, YuenKeung Hor ${ }^{20}$, Shaojing Hou ${ }^{10}$, Yee Hsiung ${ }^{40}$, Bei-Zhen $\mathrm{Hu}^{40}$, Hang $\mathrm{Hu}^{20}$, Jianrun $\mathrm{Hu}^{10}$, Jun $\mathrm{Hu}^{10}$, Shouyang $\mathrm{Hu}^{9}$, Tao $\mathrm{Hu}^{10}$, Zhuojun $\mathrm{Hu}^{20}$, Chunhao Huang ${ }^{20}$, Guihong Huang ${ }^{10}$, Hanxiong Huang ${ }^{9}$, Wenhao Huang ${ }^{25}$, Xin Huang ${ }^{10}$, Xingtao Huang ${ }^{25}$, Yongbo Huang ${ }^{28}$, Jiaqi Hui ${ }^{30}$, Lei Huo $^{21}$, Wenju Huo ${ }^{22}$, Cédric Huss ${ }^{44}$, Safeer Hussain ${ }^{66}$, Ara Ioannisian ${ }^{1}$, Roberto Isocrate ${ }^{60}$, Beatrice Jelmini ${ }^{61}$, Kuo-Lun Jen ${ }^{38}$, Ignacio Jeria ${ }^{5}$, Xiaolu Ji ${ }^{10}$, Xingzhao $\mathrm{Ji}^{20}$, Huihui Jia ${ }^{33}$, Junji Jia ${ }^{34}$, Siyu Jian ${ }^{9}$, Di Jiang ${ }^{22}$, Wei Jiang ${ }^{10}$, Xiaoshan Jiang ${ }^{10}$, Ruyi $\mathrm{Jin}^{10}$, Xiaoping Jing ${ }^{10}$, Cécile Jollet ${ }^{44}$, Jari Joutsenvaara ${ }^{42}$, Sirichok Jungthawann ${ }^{73}$, Leonidas Kalousis ${ }^{45}$, Philipp Kampmann ${ }^{50}, \mathrm{Li}$ Kang $^{18}$, Rebin Karaparambil ${ }^{47}$, Narine Kazarian ${ }^{1}$, Khanchai Khosonthongkee ${ }^{73}$, Denis Korablev ${ }^{67}$, Konstantin Kouzakov ${ }^{69}$, Alexey Krasnoperov $^{67}$, Andre Kruth ${ }^{51}$, Nikolay Kutovskiy ${ }^{67}$, Pasi Kuusiniemi ${ }^{42}$, Tobias Lachenmaier ${ }^{54}$, Cecilia Landini ${ }^{57}$, Sébastien Leblanc ${ }^{44}$, Victor Lebrin ${ }^{47}$, Frederic Lefevre ${ }^{47}$, Ruiting Lei ${ }^{18}$, Rupert Leitner ${ }^{41}$, Jason Leung ${ }^{38}$, Demin $\mathrm{Li}^{37}$, Fei Li ${ }^{10}, \mathrm{Fule} \mathrm{Li}^{13}$, Haitao $\mathrm{Li}^{20}$, Huiling $\mathrm{Li}^{10}$, Jiaqi $\mathrm{Li}^{20}$, Mengzhao $\mathrm{Li}^{10}$, Min $\mathrm{Li}^{11}$, Nan Li ${ }^{10}$, Nan $\mathrm{Li}^{16}$, Qingjiang Li ${ }^{16}$, Ruhui $\mathrm{Li}^{10}$, Shanfeng

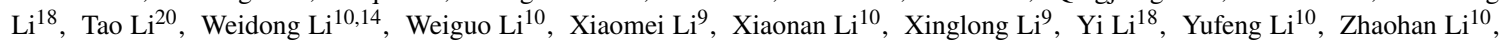
Zhibing $\mathrm{Li}^{20}$, Ziyuan $\mathrm{Li}^{20}$, Hao Liang ${ }^{9}$, Hao Liang ${ }^{22}$, Jiajun Liao ${ }^{20}$, Daniel Liebau ${ }^{51}$, Ayut Limphirat ${ }^{73}$, Sukit Limpijumnong ${ }^{73}$, Guey-Lin Lin ${ }^{38}$, Shengxin Lin ${ }^{18}$, Tao Lin ${ }^{10}$, Jiajie Ling ${ }^{20}$, Ivano Lippi60, Fang Liu ${ }^{11}$, Haidong Liu ${ }^{37}$, Hongbang Liu ${ }^{28}$, Hongjuan

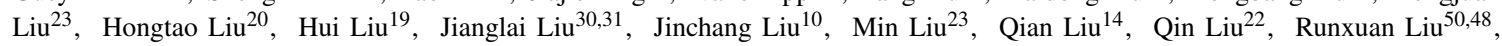
Shuangyu Liu ${ }^{10}$, Shubin Liu ${ }^{22}$, Shulin Liu ${ }^{10}$, Xiaowei Liu ${ }^{20}$, Xiwen $\mathrm{Liu}^{28}$, Yan Liu ${ }^{10}$, Yunzhe Liu ${ }^{10}$, Alexey Lokhov ${ }^{69,68}$, Paolo Lombardi ${ }^{57}$, Claudio Lombardo ${ }^{55}$, Kai Loo ${ }^{52}$, Chuan $\mathrm{Lu}^{32}$, Haoqi $\mathrm{Lu}^{10}$, Jingbin $\mathrm{Lu}^{15}$, Junguang $\mathrm{Lu}^{10}$, Shuxiang Lu ${ }^{37}$, Xiaoxu $\mathrm{Lu}^{10}$, Bayarto Lubsandorzhiev ${ }^{68}$, Sultim Lubsandorzhiev ${ }^{68}$, Livia Ludhova ${ }^{50,48}$, Arslan Lukanov ${ }^{68}$, Fengjiao Luo ${ }^{10}$, Guang Luo ${ }^{20}$, Pengwei Luo ${ }^{20}$, Shu Luo ${ }^{36}$, Wuming Luo ${ }^{10}$, Vladimir Lyashuk ${ }^{68}$, Bangzheng $\mathrm{Ma}^{25}$, Qiumei $\mathrm{Ma}^{10}, \mathrm{Si} \mathrm{Ma}^{10}$, Xiaoyan Ma ${ }^{10}$, Xubo $\mathrm{Ma}^{11}$, Jihane Maalmi ${ }^{43}$, Yury Malyshkin ${ }^{67}$, Roberto Carlos Mandujano ${ }^{67}$, Fabio Mantovani ${ }^{56}$, Francesco Manzali ${ }^{61}$, Xin Mao ${ }^{7}$, Yajun Mao ${ }^{12}$, Stefano M. Mari ${ }^{64}$, Filippo Marini ${ }^{61}$, Sadia Marium ${ }^{66}$, Cristina Martellini ${ }^{64}$, Gisele Martin-Chassard ${ }^{43}$, Agnese Martini $^{63}$, Matthias Mayer ${ }^{53}$, Davit Mayilyan ${ }^{1}$, Ints Mednieks ${ }^{65}$, Yue Meng ${ }^{30}$, Anselmo Meregaglia ${ }^{44}$, Emanuela Meroni ${ }^{57}$, David Meyhöfer $^{49}$, Mauro Mezzetto ${ }^{60}$, Jonathan Miller ${ }^{6}$, Lino Miramonti ${ }^{57}$, Paolo Montini ${ }^{64}$, Michele Montuschi ${ }^{56}$, Axel Müller ${ }^{54}$, Massimiliano Nastasi ${ }^{58}$, Dmitry V. Naumov ${ }^{67}$, Elena Naumova ${ }^{67}$, Diana Navas-Nicolas ${ }^{43}$, Igor Nemchenok ${ }^{67}$, Minh Thuan Nguyen $\mathrm{Thi}^{38}$, Feipeng Ning ${ }^{10}$, Zhe Ning ${ }^{10}$, Hiroshi Nunokawa ${ }^{4}$, Lothar Oberauer ${ }^{53}$, Juan Pedro Ochoa-Ricoux ${ }^{74,5}$, Alexander Olshevskiy ${ }^{67}$, Domizia Orestano $^{64}$, Fausto Ortica ${ }^{62}$, Rainer Othegraven ${ }^{52}$, Hsiao-Ru Pan ${ }^{40}$, Alessandro Paoloni ${ }^{63}$, Sergio Parmeggiano ${ }^{57}$, Yatian $\mathrm{Pei}^{10}$, Nicomede Pelliccia ${ }^{62}$, Anguo Peng ${ }^{23}$, Haiping Peng ${ }^{22}$, Frédéric Perrot ${ }^{44}$, Pierre-Alexandre Petitjean ${ }^{2}$, Fabrizio Petrucci ${ }^{64}$, Oliver Pilarczyk ${ }^{52}$, Luis Felipe Piñeres Rico ${ }^{45}$, Artyom Popov ${ }^{69}$, Pascal Poussot ${ }^{45}$, Wathan Pratumwan ${ }^{73}$, Ezio Previtali ${ }^{58}$, Fazhi $\mathrm{Qi}^{10}$, Ming $\mathrm{Qi}^{27}$, Sen Qian ${ }^{10}$, Xiaohui Qian ${ }^{10}$, Zhen Qian ${ }^{20}$, Hao Qiao ${ }^{12}$, Zhonghua Qin $^{10}$, Shoukang Qiu ${ }^{23}$, Muhammad Usman Rajput $^{66}$, Gioacchino Ranucci ${ }^{57}$, Neill Raper ${ }^{20}$, Alessandra Re ${ }^{57}$, Henning Rebber ${ }^{49}$, Abdel Rebii ${ }^{44}$, Bin Ren ${ }^{18}$, Jie Ren ${ }^{9}$, Barbara Ricci ${ }^{56}$, Markus Robens ${ }^{51}$, Mathieu Roche ${ }^{44}$, Narongkiat Rodphai ${ }^{71}$, Aldo Romani ${ }^{62}$, Bedřich Roskovec ${ }^{41}$, Christian Roth $^{51}$, Xiangdong Ruan ${ }^{28}$, Xichao Ruan ${ }^{9}$, Saroj Rujirawat ${ }^{73}$, Arseniy Rybnikov ${ }^{67}$, Andrey Sadovsky ${ }^{67}$, Paolo Saggese ${ }^{57}$, Simone Sanfilippo $^{64}$, Anut Sangka ${ }^{72}$, Nuanwan Sanguansak ${ }^{73}$, Utane Sawangwit ${ }^{72}$, Julia Sawatzki ${ }^{53}$, Fatma Sawy ${ }^{61}$, Michaela Schever ${ }^{50,48}$, Cédric Schwab $^{45}$, Konstantin Schweizer ${ }^{53}$, Alexandr Selyuninin, Andrea Serafini ${ }^{56}$, Giulio Settanta ${ }^{50}$, Mariangela Settimo ${ }^{47}$, Zhuang Shao ${ }^{35}$, Vladislav Sharov ${ }^{67}$, Arina Shaydurova ${ }^{67}$, Jingyan Shi ${ }^{10}$, Yanan Shi ${ }^{10}$, Vitaly Shutov ${ }^{67}$, Andrey Sidorenkov ${ }^{68}$, Fedor Šimkovic $^{70}$, Chiara Sirignano ${ }^{61}$, Jaruchit Siripak ${ }^{73}$, Monica Sisti ${ }^{58}$, Maciej Slupecki ${ }^{42}$, Mikhail Smirnov ${ }^{20}$, Oleg Smirnov ${ }^{67}$, Thiago Sogo-Bezerra ${ }^{47}$, Sergey Sokolov ${ }^{67}$, Julanan Songwadhana ${ }^{73}$, Boonrucksar Soonthornthum ${ }^{72}$, Albert Sotnikov ${ }^{67}$, Ondřej Šrámek ${ }^{41}$, Warintorn Sreethawong ${ }^{73}$, Achim Stahl ${ }^{48}$, Luca Stanco ${ }^{60}$, Konstantin Stankevich ${ }^{69}$, Dušan Štefánik ${ }^{70}$, Hans Steiger ${ }^{52,53}$, Jochen Steinmann ${ }^{48}$, Tobias Sterr ${ }^{54}$, Matthias Raphael Stock ${ }^{53}$, Virginia Strati ${ }^{56}$, Alexander Studenikin ${ }^{69}$, Shifeng Sun ${ }^{11}$, Xilei $\mathrm{Sun}^{10}$, Yongjie Sun ${ }^{22}$, Yongzhao Sun ${ }^{10}$, Narumon Suwonjandee ${ }^{71}$, Michal Szelezniak ${ }^{45}$, Jian Tang ${ }^{20}$, Qiang Tang ${ }^{20}$, Quan Tang ${ }^{23}$, 
Xiao Tang ${ }^{10}$, Alexander Tietzsch ${ }^{54}$, Igor Tkachev ${ }^{68}$, Tomas Tmej ${ }^{41}$, Marco Danilo Claudio Torri ${ }^{41}$, Konstantin Treskov ${ }^{67}$, Andrea Triossi $^{45}$, Giancarlo Troni ${ }^{5}$, Wladyslaw Trzaska ${ }^{42}$, Cristina Tuve ${ }^{55}$, Nikita Ushakov ${ }^{68}$, Johannes van den Boom ${ }^{51}$, Stefan van Waasen $^{51}$, Guillaume Vanroyen ${ }^{47}$, Vadim Vedin ${ }^{65}$, Giuseppe Verde ${ }^{55}$, Maxim Vialkov ${ }^{69}$, Benoit Viaud ${ }^{47}$, Moritz Vollbrecht ${ }^{50,48}$, Cristina Volpe ${ }^{43}$, Vit Vorobel ${ }^{41}$, Dmitriy Voronin ${ }^{68}$, Lucia Votano ${ }^{63}$, Pablo Walker ${ }^{5}$, Caishen Wang ${ }^{18}$, Chung-Hsiang Wang ${ }^{39}$, En Wang $^{37}$, Guoli Wang ${ }^{21}$, Jian Wang ${ }^{22}$, Jun Wang ${ }^{20}$, Kunyu Wang ${ }^{10}$, Lu Wang ${ }^{10}$, Meifen Wang ${ }^{10}$, Meng Wang ${ }^{23}$, Meng Wang ${ }^{25}$, Ruiguang Wang ${ }^{10}$, Siguang Wang ${ }^{12}$, Wei Wang ${ }^{27}$, Wei Wang ${ }^{20}$, Wenshuai Wang ${ }^{10}$, Xi Wang ${ }^{16}$, Xiangyue Wang ${ }^{20}$, Yangfu Wang ${ }^{10}$, Yaoguang Wang ${ }^{10}$, Yi Wang ${ }^{13}$, Yi Wang ${ }^{24}$, Yifang Wang ${ }^{10}$, Yuanqing Wang ${ }^{13}$, Yuman Wang ${ }^{27}$, Zhe Wang ${ }^{13}$, Zheng Wang ${ }^{10}$, Zhimin Wang ${ }^{10}$, Zongyi Wang ${ }^{13}$, Muhammad Waqas ${ }^{66}$, Apimook Watcharangkool ${ }^{72}$, Lianghong Wei ${ }^{10}$, Wei Wei ${ }^{10}$, Wenlu Wei ${ }^{10}$, Yadong Wei ${ }^{18}$, Kaile Wen ${ }^{10}$, Liangjian Wen ${ }^{10}$, Christopher Wiebusch ${ }^{48}$, Steven Chan-Fai Wong ${ }^{20}$, Bjoern Wonsak ${ }^{49}$, Diru Wu ${ }^{10}$, Qun $\mathrm{Wu}^{25}$, Zhi Wu ${ }^{10}$, Michael Wurm ${ }^{52}$, Jacques Wurtz ${ }^{45}$, Christian Wysotzki ${ }^{48}$, Yufei Xi ${ }^{32}$, Dongmei Xia ${ }^{17}$, Xiaochuan Xie ${ }^{17}$, Yuguang $X i^{10}$, Zhangquan $\mathrm{Xie}^{10}$, Zhizhong Xing ${ }^{10}$, Benda $\mathrm{Xu}^{13}$, Cheng $\mathrm{Xu}^{23}$, Donglian $\mathrm{Xu}^{31,30}$, Fanrong $\mathrm{Xu}^{19}$, Hangkun $\mathrm{Xu}^{10}$, Jilei $\mathrm{Xu}^{10}$, Jing $\mathrm{Xu}^{8}$, Meihang $\mathrm{Xu}^{10}$, Yin $\mathrm{Xu}^{33}$, Yu Xu${ }^{50,48}$, Baojun Yan ${ }^{10}$, Taylor Yan ${ }^{73}$, Wenqi Yan ${ }^{10}$, Xiongbo Yan ${ }^{10}$, Yupeng Yan $^{73}$, Anbo Yang ${ }^{10}$, Changgen Yang ${ }^{10}$, Chengfeng Yang ${ }^{28}$, Huan Yang ${ }^{10}$, Jie Yang ${ }^{37}$, Lei Yang ${ }^{18}$, Xiaoyu Yang ${ }^{10}$, Yifan Yang ${ }^{10}$, Yifan Yang ${ }^{2}$, Haifeng Yao ${ }^{10}$, Zafar Yasin ${ }^{66}$, Jiaxuan $\mathrm{Ye}^{10}$, Mei Ye ${ }^{10}$, Ziping Ye ${ }^{31}$, Ugur Yegin ${ }^{51}$, Frédéric Yermia ${ }^{47}$, Peihuai $\mathrm{Yi}^{10}$, Na Yin ${ }^{25}$, Xiangwei Yin ${ }^{10}$, Zhengyun You ${ }^{20}$, Boxiang $\mathrm{Yu}^{10}$, Chiye $\mathrm{Yu}^{18}$, Chunxu Yu${ }^{33}$, Hongzhao Yu${ }^{20}$, Miao $\mathrm{Yu}^{34}$, Xianghui $\mathrm{Yu}^{33}$, Zeyuan $\mathrm{Yu}^{10}$, Zezhong $\mathrm{Yu}^{10}$, Chengzhuo Yuann ${ }^{10}$, Ying Yuan ${ }^{12}$, Zhenxiong Yuan ${ }^{13}$, Ziyi Yuan ${ }^{34}$, Baobiao Yue ${ }^{20}$, Noman Zafar ${ }^{66}$, Andre Zambanini ${ }^{51}$, Vitalii Zavadskyi ${ }^{67}$, Shan Zeng ${ }^{10}$, Tingxuan Zeng ${ }^{10}$, Yuda Zeng ${ }^{20}$, Liang Zhan ${ }^{10}$, Aiqiang Zhang $^{13}$, Feiyang Zhang ${ }^{30}$, Guoqing Zhang ${ }^{10}$, Haiqiong Zhang ${ }^{10}$, Honghao Zhang ${ }^{20}$, Jiawen Zhang ${ }^{10}$, Jie Zhang ${ }^{10}$, Jin Zhang ${ }^{28}$, Jingbo Zhang ${ }^{21}$, Jinnan Zhang ${ }^{10}$, Peng Zhang ${ }^{10}$, Qingmin Zhang ${ }^{35}$, Shiqi Zhang ${ }^{20}$, Shu Zhang ${ }^{20}$, Tao Zhang ${ }^{30}$, Xiaomei Zhang ${ }^{10}$, Xuantong Zhang ${ }^{10}$, Xueyao Zhang ${ }^{25}$, Yan Zhang ${ }^{10}$, Yinhong Zhang ${ }^{10}$, Yiyu Zhang ${ }^{10}$, Yongpeng Zhang ${ }^{10}$, Yuanyuan Zhang ${ }^{30}$, Yumei Zhang ${ }^{20}$, Zhenyu Zhang ${ }^{34}$, Zhijian Zhang ${ }^{18}$, Fengyi Zhao ${ }^{26}$, Jie Zhao ${ }^{10}$, Rong Zhao ${ }^{20}$, Shujun Zhao ${ }^{37}$, Tianchi Zhao ${ }^{10}$, Dongqin Zheng ${ }^{19}$, Hua Zheng ${ }^{18}$, Minshan Zheng ${ }^{9}$, Yangheng Zheng ${ }^{14}$, Weirong Zhong ${ }^{19}$, Jing Zhou ${ }^{9}$, Li Zhou ${ }^{10}$, Nan Zhou ${ }^{22}$, Shun Zhou ${ }^{10}$, Tong Zhou ${ }^{10}$, Xiang Zhou ${ }^{34}$, Jiang Zhu ${ }^{20}$, Kangfu Zhu ${ }^{35}$, Kejun Zhu ${ }^{10}$, Zhihang Zhu ${ }^{10}$, Bo Zhuang ${ }^{10}$, Honglin Zhuang $^{10}$, Liang Zong ${ }^{13}$, and Jiaheng Zou ${ }^{10}$.

${ }^{1}$ Yerevan Physics Institute, Yerevan, Armenia.

${ }^{2}$ Université Libre de Bruxelles, Brussels, Belgium.

${ }^{3}$ Universidade Estadual de Londrina, Londrina, Brazil.

${ }^{4}$ Pontificia Universidade Catolica do Rio de Janeiro, Rio, Brazil.

${ }^{5}$ Pontificia Universidad Católica de Chile, Santiago, Chile.

${ }^{6}$ Universidad Tecnica Federico Santa Maria, Valparaiso, Chile.

${ }^{7}$ Beijing Institute of Spacecraft Environment Engineering, Beijing, China.

${ }^{8}$ Beijing Normal University, Beijing, China.

${ }^{9}$ China Institute of Atomic Energy, Beijing, China.

${ }^{10}$ Institute of High Energy Physics, Beijing, China.

${ }^{11}$ North China Electric Power University, Beijing, China.

${ }^{12}$ School of Physics, Peking University, Beijing, China.

${ }^{13}$ Tsinghua University, Beijing, China.

${ }^{14}$ University of Chinese Academy of Sciences, Beijing, China.

15 Jilin University, Changchun, China.

${ }^{16}$ College of Electronic Science and Engineering, National University of Defense Technology, Changsha, China.

${ }^{17}$ Chongqing University, Chongqing, China.

${ }^{18}$ Dongguan University of Technology, Dongguan, China.

${ }^{19}$ Jinan University, Guangzhou, China.

${ }^{20}$ Sun Yat-Sen University, Guangzhou, China.

${ }^{21}$ Harbin Institute of Technology, Harbin, China.

${ }^{22}$ University of Science and Technology of China, Hefei, China.

${ }^{23}$ The Radiochemistry and Nuclear Chemistry Group in University of South China, Hengyang, China.

${ }^{24}$ Wuyi University, Jiangmen, China.

${ }^{25}$ Shandong University, Jinan, China, and Key Laboratory of Particle Physics and Particle Irradiation of Ministry of Education, Shandong University, Qingdao, China.

${ }^{26}$ Institute of Modern Physics, Chinese Academy of Sciences, Lanzhou, China.

${ }^{27}$ Nanjing University, Nanjing, China.

${ }^{28}$ Guangxi University, Nanning, China.

${ }^{29}$ East China University of Science and Technology, Shanghai, China.

${ }^{30}$ School of Physics and Astronomy, Shanghai Jiao Tong University, Shanghai, China.

31 Tsung-Dao Lee Institute, Shanghai Jiao Tong University, Shanghai, China.

${ }^{32}$ Institute of Hydrogeology and Environmental Geology, Chinese Academy of Geological Sciences, Shijiazhuang, China.

${ }^{33}$ Nankai University, Tianjin, China.

${ }^{34}$ Wuhan University, Wuhan, China.

${ }^{35}$ Xi'an Jiaotong University, Xi'an, China.

${ }^{36}$ Xiamen University, Xiamen, China. 
${ }^{37}$ School of Physics and Microelectronics, Zhengzhou University, Zhengzhou, China.

${ }^{38}$ Institute of Physics, National Yang Ming Chiao Tung University, Hsinchu.

${ }^{39}$ National United University, Miao-Li.

${ }^{40}$ Department of Physics, National Taiwan University, Taipei.

${ }^{41}$ Charles University, Faculty of Mathematics and Physics, Prague, Czech Republic.

${ }^{42}$ University of Jyvaskyla, Department of Physics, Jyvaskyla, Finland.

${ }^{43}$ IJCLab, Université Paris-Saclay, CNRS/IN2P3, 91405 Orsay, France.

${ }^{44}$ Univ. Bordeaux, CNRS, CENBG, UMR 5797, F-33170 Gradignan, France.

45 IPHC, Université de Strasbourg, CNRS/IN2P3, F-67037 Strasbourg, France.

${ }^{46}$ Centre de Physique des Particules de Marseille, Marseille, France.

${ }^{47}$ SUBATECH, Université de Nantes, IMT Atlantique, CNRS-IN2P3, Nantes, France.

${ }^{48}$ III. Physikalisches Institut B, RWTH Aachen University, Aachen, Germany.

${ }^{49}$ Institute of Experimental Physics, University of Hamburg, Hamburg, Germany.

${ }^{50}$ Forschungszentrum Jülich GmbH, Nuclear Physics Institute IKP-2, Jülich, Germany.

${ }^{51}$ Forschungszentrum Jülich GmbH, Central Institute of Engineering, Electronics and Analytics - Electronic Systems (ZEA-2), Jülich, Germany.

52 Institute of Physics, Johannes-Gutenberg Universität Mainz, Mainz, Germany.

53 Technische Universität München, München, Germany.

${ }^{54}$ Eberhard Karls Universität Tübingen, Physikalisches Institut, Tübingen, Germany.

55 INFN Catania and Dipartimento di Fisica e Astronomia dell Università di Catania, Catania, Italy.

${ }^{56}$ Department of Physics and Earth Science, University of Ferrara and INFN Sezione di Ferrara, Ferrara, Italy.

${ }^{57}$ INFN Sezione di Milano and Dipartimento di Fisica dell Università di Milano, Milano, Italy.

${ }^{58}$ INFN Milano Bicocca and University of Milano Bicocca, Milano, Italy.

${ }^{59}$ INFN Milano Bicocca and Politecnico of Milano, Milano, Italy.

${ }^{60}$ INFN Sezione di Padova, Padova, Italy.

${ }^{61}$ Dipartimento di Fisica e Astronomia dell'Università di Padova and INFN Sezione di Padova, Padova, Italy.

62 INFN Sezione di Perugia and Dipartimento di Chimica, Biologia e Biotecnologie dell'Università di Perugia, Perugia, Italy.

${ }^{63}$ Laboratori Nazionali di Frascati dell'INFN, Roma, Italy.

${ }^{64}$ University of Roma Tre and INFN Sezione Roma Tre, Roma, Italy.

${ }^{65}$ Institute of Electronics and Computer Science, Riga, Latvia.

${ }^{66}$ Pakistan Institute of Nuclear Science and Technology, Islamabad, Pakistan.

67 Joint Institute for Nuclear Research, Dubna, Russia.

${ }^{68}$ Institute for Nuclear Research of the Russian Academy of Sciences, Moscow, Russia.

${ }^{69}$ Lomonosov Moscow State University, Moscow, Russia.

${ }^{70}$ Comenius University Bratislava, Faculty of Mathematics, Physics and Informatics, Bratislava, Slovakia.

${ }^{71}$ Department of Physics, Faculty of Science, Chulalongkorn University, Bangkok, Thailand.

${ }^{72}$ National Astronomical Research Institute of Thailand, Chiang Mai, Thailand.

${ }^{73}$ Suranaree University of Technology, Nakhon Ratchasima, Thailand.

${ }^{74}$ Department of Physics and Astronomy, University of California, Irvine, California, USA. 